\title{
Clearing Model for Day-ahead Market Considering Hydraulic Coupling Relationship of Multi-operator Cascade Hydropower Stations
}

\author{
HUANG Ningxin ${ }^{1}, J_{N}$ Z Zhaoxia ${ }^{1}$ \\ ${ }^{1}$ School of Electric Power, South China University of Technology, Guangzhou, China
}

\begin{abstract}
There is a hydraulic coupling relationship between the upstream and downstream of cascade hydropower stations, and they usually belong to different stakeholders. Traditional clearing mechanism may lead to the mismatch between the bid-winning power and the actual power generated by the downstream power stations, and the low-cost downstream power stations may lose some chance to generate, resulting in damage to social welfare. This paper analyzes the problems faced by multi-operator cascade hydropower stations participating in the day-ahead market. By deducing the generation coupling relationship between upstream and downstream cascade hydropower stations, it is found that the output of downstream hydropower stations can be divided into four parts: fixed part, adjustable part, the part coupled with the output of the upstream power station, the part coupled with the output of the upstream power station and the adjustable output. At last, day-ahead market clearing mechanism and settlement mechanism for downstream power stations to participate in bidding are proposed.
\end{abstract}

\section{Introduction}

In October 2017, the National Development and Reform Commission (NDRC) and the National Energy Administration (NEA) issued a notice on promoting the hydropower consumption in Southwest China, encouraging the development of spot electricity market pilot projects, and forming a market-oriented mechanism and price mechanism conducive to the optimal allocation of hydropower. In recent years, the scale of cascade hydropower development and utilization in China's river basins has been expanding, and the installed capacity and energy production of hydropower in Yunnan and Sichuan provinces have accounted for more than $70 \%$. However, there is still a lack of practice for cascade hydropower to participate in the spot market.

There is a hydraulic coupling relationship between the upstream and downstream power stations of cascade hydropower, which usually belong to different stakeholders and therefore, face many challenges when participating in the spot market ${ }^{[1-4]}$. For this case, literature [2-4] proposed a day-ahead market clearing model with independent participation of upstream and downstream hydropower stations. To an extent, the model can alleviate the problem that the bid-winning power of downstream hydropower stations does not match the actual power generated, resulting in water abandonment or difficult execution of trading results. However, literature [2,3] regards downstream power stations as price takers, which limits the autonomy of stakeholders of downstream power stations to participate in the market. In literature [4], the clearing method of mandatory acquisition of downstream power stations' generating capacity in dry season may lead to problems such as higher cost of purchasing electricity in the market.

This paper summarizes and analyzes the difficulties of multi-operator cascade hydropower participating in the day-ahead market, deduces the generation coupling relationship between upstream and downstream power stations, and the adjustable output of downstream stations can participate in the market quotation. At last, we put forward the corresponding quotation mechanism, clearing model and settlement mechanism of day-ahead market for downstream power stations to participate in.

\section{Problems in the traditional day-ahead market clearing model}

a) The generation coupling relationship of upstream and downstream power stations is not considered

The output of the downstream power station is affected by the upstream power station. Only when the bid-winning power of the upstream power station is determined, the relationship of output and generation flow of the downstream power station can be determined ${ }^{[2]}$. However, the traditional dispatching model does not consider the generation coupling relationship of upstream and downstream power stations, which may lead to the failure of downstream power stations to execute the bid-winning power. If the downstream power stations do not participate in the market quotation or offer zero price, it is not in line with

\footnotetext{
* Corresponding author: jxxghnx901@qq.com
} 
the interests of the downstream power stations that have certain capacity regulation ability.

b) Low-cost downstream power stations may lose some power generation opportunities

In addition to the generation coupling relationship, the revenue of downstream power stations mainly depends on market clearing price and the bid-winning power of upstream power stations. For the case that the upstream and downstream power stations belong to the same stakeholder, the producer will set the quotation strategy with the goal of maximizing the total profit of the cascade power stations. However, for the case that the upstream and downstream power stations belong to different stakeholders, the upstream power station will make the quotation with the goal of maximizing its own profit. This may cause the low-cost downstream power stations to lose some opportunities to generate due to limited water supply, reduce social welfare, and is not conducive to the optimal allocation of resources.

\section{Hydraulic coupling relationship of cascade hydropower stations}

\subsection{Water quantity coupling relationship of cascade upstream and downstream hydropower stations}

Figure 1 is a schematic diagram of cascade hydropower stations in a basin. For hydropower station $i$, water balance constraints should be met:

$$
\begin{aligned}
& V_{i, t}-V_{i, t-1}=\left(Q_{i, t}^{i n}-Q_{i, t}^{H}-Q_{i, t, L}^{H}\right) \Delta t \\
& Q_{i, t}^{i n}=I_{i, t}+\alpha e^{\gamma}\left(Q_{k, t-\tau}^{H}+Q_{k, t-\tau, L}^{H}\right)
\end{aligned}
$$

Where: $V_{i, t}$ is the storage capacity; $Q_{i, t}^{i n}, Q_{i, t}^{H}$ and $Q_{i, t, L}^{H}$ are the inflow, generation flow and abandoned water flow respectively; the inflow of the section depends on the natural inflow, generation flow and the abandoned water flow of the upstream power station $k$ at time $t-\tau$; $\tau$ is the hydraulic time lag between the upstream power station $k$ and the downstream power station $i ; \alpha$ is the diversion coefficient of the river channel; $e^{\gamma}$ is the channel smoothing coefficient.

The regulation of reservoir capacity will cause the change of reservoir water level, and the relationship between them can be approximately linear (Eq. (3), where $a_{1}$ and $b_{1}$ are non-zero constants).

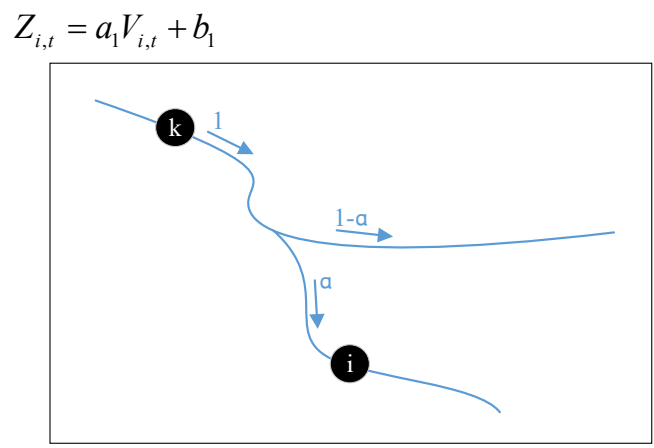

Figure 1. Schematic diagram of cascade hydropower in a certain basin
3.2 Generation coupling relationship of cascade upstream and downstream hydropower stations

\subsubsection{Hydropower Modeling}

Generally speaking, the output of hydropower station $i$ at time $t$ can be expressed as:

$$
\begin{aligned}
& P_{i, t}=9.81 \times \eta_{i} H_{i, t} Q_{i, t}^{H} \\
& H_{i, t}=\frac{Z_{i, t}+Z_{i, t-1}}{2}-Z_{i, t}^{d}-H_{i, t}^{d}
\end{aligned}
$$

Where: $\eta_{i}$ is the power generation efficiency of hydropower station $i$; $H_{i, t}$ is the working head; $Z_{i, t}^{d}$ is the tail water level and $H_{i, t}^{d}$ is the head loss. In order to ensure the maximum benefit of water resources, the abandoned water flow of each hydropower station is $\operatorname{zero}\left(Q_{i, t, L}^{H}=0\right)$ in normal water period and period of low flow. Therefore, equation (1) can be changed into equation (6), and the tail water level and head loss of hydropower station $i$ can also be approximately expressed as first-order functions of generation flow ${ }^{[2]}$ (Eq.(7) and (8)). Where, $a_{Z Q, i}$ and $b_{z Q, i}$ are the correlation coefficients of tail water level and discharge flow; $a_{Q d, i}$ and $b_{Q d, i}$ are the correlation coefficients of head loss and discharge flow. All of them are constants.

$$
\begin{aligned}
& V_{i, t-1}=V_{i, t}-\left(Q_{i, t}^{i n}-Q_{i, t}^{H}\right) \Delta t \\
& Z_{i, t}^{d}=a_{Z Q, i} Q_{i, t}^{H}+b_{Z Q, i} \\
& H_{i, t}^{d}=a_{Q d, i} Q_{i, t}^{H}+b_{Q d, i}
\end{aligned}
$$

From equation (3) and equation (5)-(8), the working head of hydropower station $i$ can be deduced:

$$
\begin{aligned}
H_{i, t}= & \left(a_{1} V_{i, t}-\frac{a_{1} \Delta t}{2} Q_{i, t}^{i n}+b_{1}-b_{z Q, i}-b_{Q d, i}\right)+ \\
& \left(\frac{a_{1} \Delta t}{2}-a_{Z Q, i}-a_{Q d, i}\right) Q_{i, t}^{H}
\end{aligned}
$$

By substituting equation (9) into equation (4), the output of downstream power station can be obtained:

$$
\begin{aligned}
P_{i, t}= & 9.81 \eta_{i}\left[\left(a_{1} V_{i, t}-\frac{a_{1} \Delta t}{2} Q_{i, t}^{i n}+b_{1}-b_{Z Q, i}-b_{Q d, i}\right) Q_{i, t}^{H}\right. \\
& \left.+\left(\frac{a_{1} \Delta t}{2}-a_{Z Q, i}-a_{Q d, i}\right)\left(Q_{i, t}^{H}\right)^{2}\right]
\end{aligned}
$$

Similarly, the output of upstream power station can be expressed as:

$$
\begin{aligned}
P_{k, t}= & 9.81 \eta_{k}\left[\left(a_{2} V_{k, t}-\frac{a_{2} \Delta t}{2} Q_{k, t}^{i n}+b_{2}-b_{Z Q, k}-b_{Q d, k}\right) Q_{k, t}^{H}\right. \\
& \left.+\left(\frac{a_{2} \Delta t}{2}-a_{Z Q, k}-a_{Q d, k}\right)\left(Q_{k, t}^{H}\right)^{2}\right]
\end{aligned}
$$

It can be seen that the output of hydropower station at time $t$ is related to the reservoir capacity, water inflow and generation flow at that time.

\subsubsection{Relationship of output and generation flow of the upstream power station}

The water inflow of upstream power station is usually stable, suppose $Q_{k, t}^{i n}=C$ and the initial storage capacity 
of the upstream reservoir is $V_{k, t_{0}}$, the storage capacity at time $t$ can be expressed as:

$$
V_{k, t}=V_{k, t_{0}}+\int_{t_{0}}^{t}\left(C-Q_{k, t}^{H}\right) d t
$$

By substituting equation (12) into equation (11), we get the following results:

$$
\begin{aligned}
& P_{k, t}=9.81 \eta_{k}\left[\left(a_{2} V_{k, t_{0}}+a_{2} \int_{t_{0}}^{t}\left(C-Q_{k, t}^{H}\right) d t-\frac{a_{2} \Delta t}{2} C+\right.\right. \\
& \left.\left.b_{2}-b_{Z Q, k}-b_{Q d, k}\right) Q_{k, t}^{H}+\left(\frac{a_{2} \Delta t}{2}-a_{Z Q, k}-a_{Q d, k}\right)\left(Q_{k, t}^{H}\right)^{2}\right]
\end{aligned}
$$

It can be seen that the output of the upstream power station is a nonlinear function of the generation flow. In order to simplify the calculation, the piecewise linear function (Eq.(14)) is obtained by piecewise linear fitting of the equation (13).

$$
P_{k, t}=a_{k . t}^{s_{k}} Q_{k, t}^{H}+b_{k . t}^{s_{k}}
$$

Where: $s_{k}$ is the segment number; $a_{k . t}^{s_{k}}$ and $b_{k . t}^{s_{k}}$ are respectively the fitting coefficient of the linear term and constant term of $s_{k}$ segment, which are related to the power generation efficiency, reservoir capacity, the correlation coefficients of tail water level and discharge flow and the correlation coefficients of head loss and discharge flow.

\subsection{3 generation coupling relationship of upstream and downstream power stations}

The inflow of downstream power station includes natural inflow and generation flow of upstream power station. Assuming that the natural inflow of downstream power station is stable $\left(I_{i, t}=c\right)$ and the initial storage capacity is $V_{i, t_{0}}$, then the storage capacity at time $t$ can be expressed as:

$$
V_{i, t}=V_{i, t_{0}}+\int_{t_{0}}^{t}\left(c+\alpha e^{\gamma} Q_{k, t-\tau}^{H}-Q_{i, t}^{H}\right) d t
$$

By substituting equation (15) into equation (10), we get the following results:

$$
\begin{aligned}
P_{i, t}= & P_{i, t}^{\prime}+P_{i, t}^{\prime \prime} \\
P_{i, t}^{\prime} & =9.81 \eta_{i}\left[\left(a_{1} V_{i, t_{0}}-a_{1} \int_{t_{0}}^{t} Q_{i, t}^{H} d t+b_{1}-\right.\right. \\
b_{Z Q, i} & \left.\left.-b_{Q d, i}\right) Q_{i, t}^{H}+\left(\frac{a_{1} \Delta t}{2}-a_{z Q, i}-a_{Q d, i}\right)\left(Q_{i, t}^{H}\right)^{2}\right] \\
P_{i, t}^{\prime \prime}= & 9.81 \eta_{i}\left[a_{1} \int_{t_{0}}^{t}\left(c+\alpha e^{\gamma} Q_{k, t-\tau}^{H}\right) d t\right. \\
& \left.-\frac{a_{1} \Delta t}{2}\left(c+\alpha e^{\gamma} Q_{k, t-\tau}^{H}\right)\right] Q_{i, t}^{H}
\end{aligned}
$$

It can be seen that the output of downstream power station is not only related to its own generation flow, but also depends on the generation flow of upstream power station. In order to simplify the calculation, the piecewise linear functions (Eq.(19) and (20)) is used to fit equations (17) (18).

$$
\begin{aligned}
& P_{i, t}^{\prime}=a_{i . t}^{s_{i}^{\prime}} Q_{i, t}^{H}+b_{i . t}^{s_{i}^{\prime}} \\
& P_{i, t}^{\prime \prime}=\left(a_{i . t}^{s_{i}^{\prime}} Q_{k, t-\tau}^{H}+b_{i . t}^{s_{i}^{\prime \prime}}\right) Q_{i, t}^{H}
\end{aligned}
$$

Where: $s_{i}^{\prime}$ and $s_{i}^{\prime \prime}$ are the segment numbers; $a_{i . t}^{s_{i}^{\prime}}$ and $b_{i . t}^{s_{i}^{\prime}}$ are respectively the fitting coefficients of the linear term and constant term of the $s_{i}^{\prime}$ segment, which are related to the power generation efficiency, reservoir capacity, the correlation coefficients of tail water level and discharge flow and the correlation coefficients of head loss and discharge flow; $a_{i . t}^{s_{i}^{*}}$ and $b_{i . t}^{s_{i}^{*}}$ are respectively the fitting coefficients of the linear term and constant term of the $s_{i}$ " segment, which are determined by the power generation efficiency of the downstream power station, the diversion coefficient of the river channel and the smoothing coefficient.

By substituting formula (14) and formula (19)-(20) into formula (16), we get the following results:

$$
P_{i, t}=\alpha_{t}+\beta_{t} P_{i, t}^{\prime}+\gamma_{t} P_{k, t-\tau}+\varphi_{t} P_{i, t}^{\prime} P_{k, t-\tau}
$$

Where:

$$
\left\{\begin{array}{l}
\alpha_{t}=\frac{b_{i . t}^{s_{i}} a_{i . t}^{s_{i}^{\prime \prime}} b_{k . t}^{s_{k}}}{a_{i . t}^{s_{i}^{\prime}} a_{k . t}^{s_{k}}}-\frac{b_{i . t}^{s_{i}} b_{i . t}^{s_{i}^{\prime \prime}}}{a_{i . t}^{s_{i}^{\prime}}}, \quad \beta_{t}=1+\frac{b_{i . t}^{s_{i}^{\prime \prime}}}{a_{i . t}^{s_{i}^{\prime}}}-\frac{a_{i . t}^{s_{i}} b_{k . t}^{s_{k}}}{a_{i . t}^{s_{i}^{\prime}} a_{k . t}^{s_{k}}} \\
\gamma_{t}=-\frac{a_{i . t}^{s^{\prime}} b_{i . t}^{s_{i}^{\prime}}}{a_{i . t}^{s_{i}} a_{k . t}^{s_{k}}}, \quad \varphi_{t}=\frac{a_{i . t}^{s_{i}^{\prime \prime}}}{a_{i . t}^{s_{i}} a_{k . t}^{s_{k}}}
\end{array}\right.
$$

\section{Clearing mechanism of day-ahead market with Cascade Hydropower Participation}

\subsection{Quotation mechanism}

For the two-stage cascade hydropower shown in Figure 1, according to equation (21), the output of the downstream hydropower station is composed of four parts: the fixed part, the adjustable part, the part coupled with the output of the upstream hydropower station, and the part coupled with the output of upstream hydropower station and the adjustable output. The coupling relationship of each part is shown in Figure 2.

The upstream and downstream power stations need to declare the unit output constraint, water level constraint, generation flow constraint and initial storage capacity or water level. The market operator determines the relationship of output and generation flow of the upstream and downstream power stations according to the inflow forecast information and the initial storage capacity of the power stations, deduces the coefficients $\alpha_{t}, \beta_{t}, \gamma_{t}$ and $\varphi_{t}$, and publishes them to the downstream power stations. The coefficients, $\alpha_{t}, \beta_{t}, \gamma_{t}$ and $\varphi_{t}$, may be positive or negative. For the upstream power station, it is necessary to quote in the day-ahead market, while the downstream power station only needs to quote for the adjustable part, and the other three parts are the price takers, so there is no need to quote. 


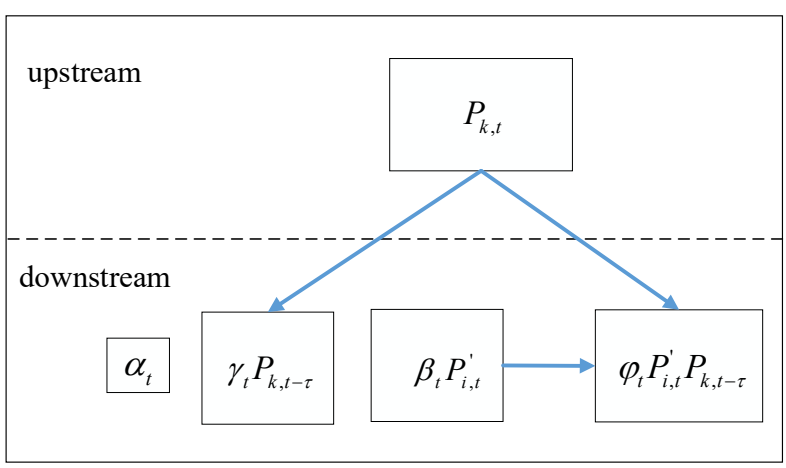

Figure 2. generation coupling relationship of upstream and downstream power stations

\subsection{Clearing model for day-ahead market}

The clearing model for day-ahead market considering the generation coupling relationship of cascade hydropower stations takes the maximum social welfare (that is, the minimum difference between generation cost and electricity benefit) as the optimization objective ${ }^{[5]}$, and considers the constraints of power balance and bidwinning power of market participants to calculate the bid-winning situation and market clearing prices.

$\min \sum_{t \in T} \sum_{s \in S} x_{s}^{t} Q_{s}^{t} P_{s}^{t}$

s.t.

$$
\begin{aligned}
& \sum_{s \in S} x_{s}^{t} Q_{s}^{t}+\sum_{m \in M}\left(\begin{array}{c}
\gamma_{t, m} \sum_{s \in S_{m, k}} x_{s}^{t-\tau} Q_{s}^{t-\tau}+ \\
\frac{\gamma_{t, m}}{\beta_{t, m}} \sum_{s \in S_{m, i}} x_{s}^{t} Q_{s}^{t} \sum_{s \in S_{m, k}} x_{s}^{t-\tau} Q_{s}^{t-\tau}
\end{array}\right)=0 \\
& 0 \leq x_{s}^{t} \leq 1 \quad \forall t \in T, s \in S
\end{aligned}
$$

Where: $t$ is the period, $T$ is the period set; $s$ is the electricity price segment, $S$ is the electricity price segment set; $S_{m, k}$ is the electricity price segment set declared by upstream hydropower station of cascade hydropower station $m ; S_{m, i}$ is the electricity price segment set declared by downstream hydropower station of cascade hydropower station $m ; M$ is the cascade hydropower station set; $x$ is the acceptance rate, i.e. the ratio of bid-winning power to declared quantity, $x=0$ means losing the bid, $x=1$ means fully winning the bid, and $x$ between 0 and 1 means partially winning the bid; $Q$ and $P$ are respectively the declared quantity and price of market participants; for the bid of buying, the quantity $Q$ is negative and the price $P$ is positive, and for the bid of selling, both the quantity $Q$ and the price $P$ are positive.

\subsection{Settlement mechanism}

If the upstream and downstream power stations belong to different stakeholders, the settlement should be made according to "bid-winning volume $\times$ market clearing price" when both the upstream and downstream power stations are profitable. When the upstream power station is at a loss and the downstream power station is at a profit, the clearing of the upstream power station whose bid price is higher than market clearing price increases the profit of the downstream power station, and the increased profit is sufficient to compensate the loss of the upstream power station (increasing overall social welfare). Therefore, the downstream power station should compensate for the cost deficiency of the upstream power station.

\section{Conclusion}

This paper analyzes the difficulities of cascade hydropower participating in day-ahead market, deduces the generation coupling relationship between upstream and downstream hydropower stations, and puts forward the corresponding day-ahead market clearing mechanism. The analysis shows that the output of the adjustable part of the downstream power station can participate in the market quotation, and the coupling part is regarded as the market price receiver, so as to avoid the problem that bid-winning power of the downstream power station cannot be executed. Due to the coupling of output, there is interest coupling between upstream and downstream hydropower stations. In order to promote the maximization of social welfare, there is bound to be a situation of sacrificing part of the benefits of upstream power stations in exchange for greater benefits generated by the corresponding coupling parts of downstream power stations. Therefore, this paper proposes that for cascade hydropower belonging to different stakeholders, income redistribution is necessary to ensure no loss incurred to upstream power station.

\section{References}

1. LU Y., HU X.J., SUN Y., et al. Design and Practice of Electricity Spot Market Mechanism Adapting to Characteristics of High Proportional Hydropower in Sichuan Province. Automation of Electric Power Systems, 1-9(in Chinese) (2021).

2. Zhang L.Z., Liu F., Xu T., et al. Clearing model for day-ahead market with participation of multioperator cascade hydropower stations. Automation of Electric Power Systems, 42(16):104-110 (in Chinese) (2018).

3. Zhang L.Z., Liu F., Xu T., et al. Self-scheduling bidding strategy for downstream station in dayahead market with participation of multi-operator cascade hydropower stations. Automation of Electric Power Systems, 42(19):27-33 (in Chinese) (2018).

4. PENG Y.X., LIU J.C., HAN X.Y., et al. Staging Clearing for Day-ahead Market Considering Multiowner Cascade Hydropower Stations. Power Systems Technology, 44(08):2837-2848 (in Chinese) (2020).

5. Zhang X.Y., Chen Q.X., Ge R., et al. Clearing model of electricity spot market considering flexible block orders. Automation of Electric Power Systems, 41(24):35-41(in Chinese) (2017). 